\title{
Method of an enhanced self-amplified spontaneous emission for x-ray free electron lasers
}

\author{
Alexander A. Zholents \\ Lawrence Berkeley National Laboratory, University of California, Berkeley, California 94720, USA
}

(Received 21 May 2004; published 12 April 2005)

\begin{abstract}
We describe a technique by which an energy modulation of electrons via interaction with a laser pulse in a wiggler magnet is used for a significant increase of the electron peak current prior to entering a long self-amplified spontaneous emission (SASE) free electron laser undulator. This results in a reduction of the gain length for the SASE process and a modification of the structure of the output $\mathrm{x}$-ray radiation. It also temporally links the output $\mathrm{x}$-ray pulse to the initial laser pulse, thus providing an opportunity for accurate synchronization between the laser pump pulse and $\mathrm{x}$-ray probe pulse for pump-probe experiments.
\end{abstract}

DOI: 10.1103/PhysRevSTAB.8.040701

The technique of self-amplified spontaneous emission (SASE) (see [1] and references therein) is a widely acknowledged tool for production of an intense flux of photons with several $\mathrm{keV}$ photon energies. Two x-ray SASE free electron laser (FEL) projects are already in an advanced stage [2]. In this paper we propose a significant enhancement of the electron peak current entering the SASE FEL by using a conventional optical laser and demonstrate that this enhancement leads to a considerable reduction of the FEL gain length. The timing and duration of the x-ray pulse is also controlled by the timing and duration of the optical laser pulse, which makes pumpprobe experiments available to the SASE FEL with potential for absolute temporal synchronization. Recent success in the production of extreme ultraviolet pulses of attosecond duration at $97 \mathrm{eV}$ photon energy [3-5] has inspired various proposals for a production of (i) soft and hard x-ray attosecond pulses using ponderomotive laser acceleration [6] or SASE and harmonic cascade FELs [7-9], and (ii) attosecond electron pulses [10]. With our scheme we could also produce a solitary few hundred attosecond x-ray pulse.

Figure 1 shows a schematic of the proposed technique, which we call enhanced SASE (ESASE). On the left the electron beam passes the linac and enters a wiggler magnet. At the same time a short $\sim 50 \mathrm{fsec}$ optical laser pulse enters the wiggler and propagates through the wiggler collinearly with the electrons. The wiggler period $\lambda_{w}$ and wiggler parameter $K_{w}=e B_{w} \lambda_{w} /(2 \pi m c)$, where $m$, $e$ are the electron mass and charge, $c$ is the speed of light, and $B_{w}$ is the peak magnetic field, are chosen such that $\lambda_{L}=$ $\lambda_{w}\left(1+K_{w}^{2} / 2\right) / 2 \gamma_{w}^{2}$, where $\lambda_{L}$ is the laser wavelength and $\gamma_{w}$ is the relativistic factor for the average electron beam energy in the wiggler. The laser pulse overlaps only a short longitudinal section of the electron beam in the wiggler. For convenience we call this section the working section (WS). Electrons in the WS interact with the laser field and emerge from the wiggler with an energy modulation. The peak power of the laser field is chosen such that the amplitude of energy modulation significantly exceeds
PACS numbers: 41.50.+h, 41.60.Cr, 42.55.Vc, 52.59.-f

the uncorrelated energy spread of the electrons $\sigma_{\gamma 0}$. Next, the electron beam enters a second linear accelerator and gains energy to reach the final relativistic factor $\gamma_{x}$. This acceleration does not affect the energy modulation introduced in the wiggler and does not produce noticeable relative longitudinal motion of electrons because of the ultrarelativistic electron energies. Following acceleration the electron beam passes through a dispersive magnetic chicane where higher energy electrons travel a shorter path and lower energy electrons travel a longer path. This produces microbunching of the electrons in the WS and periodic enhancement of the electron peak current. As an illustration, Fig. 2(a) shows the longitudinal phase space of the electrons after the chicane in a short segment of one laser wavelength.

Finally, electrons enter a long undulator with period $\lambda_{u}$ and undulator parameter $K_{x}=e B_{x} \lambda_{u} /(2 \pi m c)$, where $B_{x}$ is the peak magnetic field, matched to produce radiation at the x-ray wavelength $\lambda_{x}=\lambda_{u}\left(1+K_{x}^{2} / 2\right) / 2 \gamma_{x}^{2}$ via the standard SASE process.

To simplify the analysis of the above-described scheme we consider a rectangular optical laser pulse with a peak power $P_{L}$. Generalization to a Gaussian pulse shape is straightforward. The amplitude of the electron energy gain/loss in the wiggler $\Delta \gamma_{w}$ can be calculated by integration of electron interaction with the laser field along its trajectory in the wiggler. When the Rayleigh length of the laser beam equals approximately one quarter length of the wiggler and the electron beam size including wiggles of the electron beam trajectory is smaller than the laser beam spot size at the focus, then [11]

$$
\Delta \gamma_{w}^{2}=33 \pi \frac{P_{L}}{P_{A}} N_{w} \xi_{w}\left[J_{0}\left(\xi_{w} / 2\right)-J_{1}\left(\xi_{w} / 2\right)\right]^{2},
$$

where $P_{A}=I_{A} m c^{2} / e \approx 8.7 \mathrm{GW}, I_{A}=17 \mathrm{kA}$ is the Alfvén current, $\xi_{w}=K_{w}^{2} /\left(2+K_{w}^{2}\right)$, and $J_{0}, J_{1}$ are the zero and first order Bessel functions of the first kind. $N_{w}$ is the number of wiggler periods chosen to be either smaller than the number of cycles in the laser pulse, or 


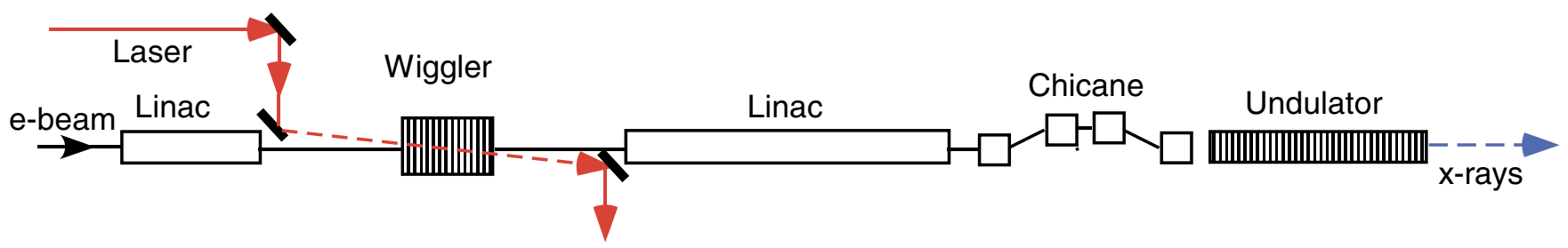

FIG. 1. (Color) A schematic of ESASE x-ray FEL.

such as to comply with an approximate condition of validity for the above formula, namely $N_{w} \Delta \gamma_{w} / \gamma_{w} \leq 0.05$.

Following [12], the standard 1D FEL particle equations in the zero gain limit may be written as

$$
\frac{d \nu}{d s_{*}}=-\Omega^{2} \sin \theta \quad \text { and } \quad \frac{d \theta}{d s_{*}}=2 \pi \nu,
$$

where $s_{*} \equiv s / L_{u}$ is the dimensionless length along the undulator, $\theta$ is the electron phase relative to the ponderomotive well, $\nu \equiv 2 N_{w}\left(\gamma-\gamma_{w}\right) / \gamma_{w}, \quad$ and $\quad \Omega^{2} \equiv$ $2 N_{w} \Delta \gamma_{w} / \gamma_{w}$ is the FEL-equivalent synchrotron tune. Using perturbation expansion of $\nu$ and $\theta$ in powers of $\Omega^{2}<1$, one obtains a solution of Eq. (2) at the end of the undulator $\left(s_{*}=1\right)$ through order $\Omega^{2}$ (see also $[8,12]$ ):

$$
\begin{aligned}
\nu_{f}= & \nu_{0}+\frac{\Omega^{2}}{2 \pi \nu_{0}}\left[\cos \left(\theta_{0}+2 \pi \nu_{0}\right)-\cos \theta_{0}\right], \\
\theta_{f}= & \theta_{0}+2 \pi \nu_{0}+\frac{\Omega^{2}}{\nu_{0}}\left[\frac{\sin \left(\theta_{0}+2 \pi \nu_{0}\right)-\sin \theta_{0}}{2 \pi \nu_{0}}\right. \\
& \left.-\cos \theta_{0}\right]
\end{aligned}
$$

where $\nu_{0}$ and $\theta_{0}$ refer to values at the wiggler entrance.

Following interaction with the laser beam in the wiggler, the electron beam is accelerated to energy $\gamma_{x}$ and passes through a magnetic chicane with a time-of-flight parameter $R_{56}$. We neglect relative longitudinal motions of the electrons during the acceleration, but consider them in the

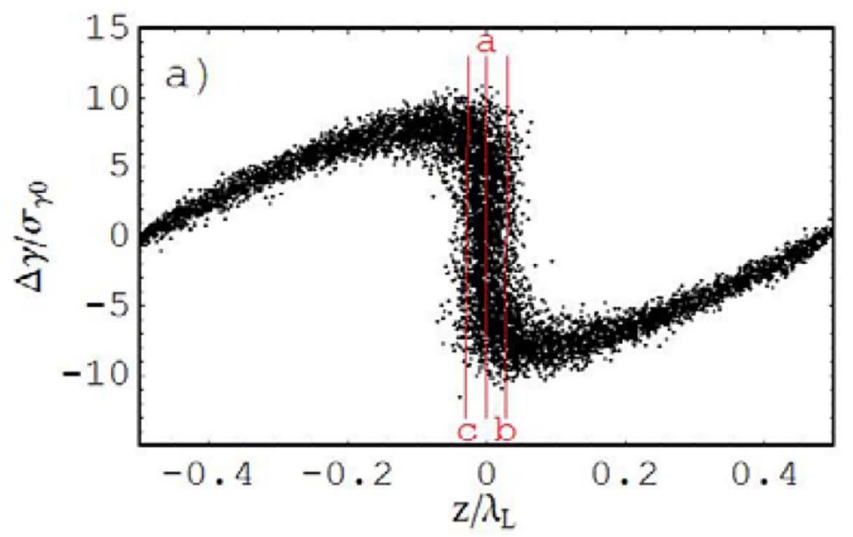

chicane. Although the laser is not present at this point it is still convenient to use the electron phase relative to ponderomotive well as a definition of electron location in the longitudinal direction. A new phase can be found from the following equation (see also [8]):

$$
\begin{aligned}
\theta_{e}= & \theta_{f}+2 \pi \alpha \nu_{f} \\
= & \theta_{0}+2 \pi \nu_{0}(\alpha+1)+C-\pi \Omega^{2}\left[(2 \alpha+1) \frac{\sin \pi \nu_{0}}{\pi \nu_{0}}\right. \\
& \left.\times \sin \tilde{\theta}-\frac{1}{\pi \nu_{0}}\left(\frac{\sin \pi \nu_{0}}{\pi \nu_{0}}-\cos \pi \nu_{0}\right) \cos \tilde{\theta}\right],
\end{aligned}
$$

where $\tilde{\theta} \equiv \theta_{0}+\pi \nu_{0}, \alpha \equiv R_{56} \gamma_{w} /\left(2 N_{w} \lambda_{L} \gamma_{x}\right)$, and $C$ is an energy independent path-length difference term. Using (4) and presuming a uniform distribution of electrons in $\theta_{0}$ and a Gaussian distribution in $\nu_{0}$ with rms value $\sigma_{\nu} \equiv$ $2 N_{w} \sigma_{\gamma 0} / \gamma_{w}$, one obtains the following expression for the WS electron current after the chicane (see also [13]):

$$
\begin{aligned}
I(z) / I_{0}= & 1+2 \sum_{n=1}^{\infty} J_{n}\left(n \pi \Omega^{2}(2 \alpha\right. \\
& +1)) \mathrm{e}^{-\left[n \pi \Omega^{2}(2 \alpha+1) / B\right]^{2} / 2} \cos (n k z),
\end{aligned}
$$

where $z$ is the coordinate along the electron bunch within the WS, $I_{0}$ is the electron current outside of the WS, $B=$ $\Delta \gamma_{w} / \sigma_{\gamma 0}$, and $k=2 \pi / \lambda_{L}$. Note that high harmonics with $n>n_{c}=\sqrt{2} B / \pi \Omega^{2}(2 \alpha+1)$ do not contribute signifi-

FIG. 2. (Color) (a) Longitudinal phase space after the chicane showing microbunching of electrons and an enhanced electron density. Only a part of the WS equivalent to one optical cycle at the laser wavelength is actually shown. (b) The histograms of the energy distribution of electrons after the chicane taken along the lines $a, b, c$ in (a). 
cantly to the sum in (5). For $n \gg 1, J_{n}(n x)$ reaches maximum at $x \approx 1+0.809 n^{-2 / 3}$ [14]. We use this fact and the above definition for $n_{c}$ to obtain an empirical value for $\pi \Omega^{2}(2 \alpha+1) \approx 1+1 /(4+B / 4)$ that maximizes the value of $I(0) / I_{0}$. The right-hand side of Eq. (5) can be accurately represented by a periodic series of the identical microbunches spaced at $\lambda_{L}$ :

$$
\begin{aligned}
I(z) / I_{0} & =\sum_{j} \mathrm{e} a B /\left\{1+\left[4 B\left(z / \lambda_{L}-j\right)\right]^{2}\right\} \\
\text { with } a & =1 /\left(1+B^{1 / \mathrm{e}}\right),
\end{aligned}
$$

where $\mathrm{e}=2.718$ is the base of the natural logarithm (not to be confused with a symbol $e$ reserved for the electron charge). Each microbunch in (6) has width $\Delta z_{0}=\lambda_{L} / 2 B$.

In the following analysis we consider only the central microbunch located within the distance $|z|<\lambda_{L} / 2$. However, all conclusions drawn for this microbunch are applicable to the entire WS because of the identical nature of all microbunches.

The increase in the peak current in the WS is accompanied by a corresponding increase in the energy spread of electrons. Figure 2(b) shows the energy distribution of electrons taken at three positions along the compressed microbunch, marked as $a, b, c$ in Fig. 2(a). Although the distribution functions in Fig. 2(b) are not Gaussian, we still use their rms values to characterize the variation of the uncorrelated energy spread of electrons as a function of $z$ near the current peak in the WS using the following empirical formula:

$$
\begin{aligned}
\sigma_{\gamma}(z) / \sigma_{\gamma 0}= & 1+a B\left\{\operatorname{erf}\left(4 z /\left(\Delta z_{0} B^{1 / 8}\right)+\pi\right)\right. \\
& \left.-\operatorname{erf}\left(4 z /\left(\Delta z_{0} B^{1 / 8}\right)-\pi\right)\right\},
\end{aligned}
$$

where erf(x) is the error function and $|z|<\lambda_{L} / 2$.

Finally, the electron beam goes through a long undulator where electrons inside the WS produce enhanced SASE because of the current enhancement. The electrons outside of the WS also produce SASE, but have a much longer gain length because of their smaller current. We show later that it takes $\sim 8$ amplitude gain lengths for SASE to reach saturation, i.e., approximately $8 \hat{M}_{G}$ undulator periods, where $\hat{M}_{G}$ is the gain length expressed in the number of undulator periods at the maximum of the current peak defined by Eq. (6). Thus, to avoid gain degradation due to the slippage of electrons relative to the light in the undulator, the microbunch in the WS should have sufficient length to support SASE until the very last gain length, i.e., $\Delta z_{0} \geq 8 \hat{M}_{G} \lambda_{x}$. This condition constrains the choice of the optical laser wavelength for a given $\lambda_{x}: \lambda_{L} / \lambda_{x} \geq 16 \hat{M}_{G} B$.

Because of the microbunched structure of the electron beam, the x-ray signal appears as a series of $N_{w}$ x-ray micropulses separated by $\lambda_{L}$. The upper estimate for the FWHM duration of each $\mathrm{x}$-ray micropulse at saturation can be written as

$$
\Delta \tau \approx\left(\Delta z_{0} \sqrt{3 \ln (2) / 16}+\hat{M}_{G} \lambda_{x}\right) / c \approx 4 \hat{M}_{G} \lambda_{x} / c,
$$

where the first term on the right-hand side shows narrowing of the x-ray pulse over the eight gain lengths caused by the variation of the electron peak current within the electron microbunch, and the second term shows broadening of the $\mathrm{x}$-ray pulse due to electron slippage relative to the light in the undulator.

We recall that the SASE output from a microbunch with a finite length $\Delta z_{0}$ becomes nearly temporally coherent and Fourier transform limited when $\Delta z_{0} \leq 2 \pi \hat{M}_{G} \lambda_{x}$ as estimated in [15]. This is remarkably close to the lower limit for the required microbunch length defined by slippage. Thus, it is reasonable to expect in our scheme to have a nearly temporally coherent and Fourier transform limited $\mathrm{x}$-ray micropulse produced by each individual microbunch. However, because of the SASE process, the carrier phase of the x-ray wave is not stable and changes randomly from one micropulse to the next.

The X-ray radiation produced by electrons outside of the WS has significantly less intensity because of the longer gain length at a significantly lower peak current. Thus, there is absolute synchronization between the chain of the output x-ray micropulses and the laser pulse since only electrons from the WS, i.e., from the region that experienced interaction with the laser, produce intense $x$ rays. This feature can be used in pump-probe experiments with the $\mathrm{x}$-ray pulse being a probe and a laser pulse or any other signal derived from laser pulse being a pump source. Moreover, as soon as the laser pulse is shorter than the electron bunch, then by changing the duration of the laser pulse and adjusting the number of active wiggler periods one can regulate the length of the WS and therefore the duration of the x-ray output. We will return to this feature later in a discussion of the generation of intense attosecond x-ray pulses.

For illustration of the above described technique we present calculations made for a hypothetical x-ray FEL. We use the electron beam parameters that are similar to what can be found in [2]: final beam energy of $14.35 \mathrm{GeV}$, normalized emittance of $1.2 \mathrm{~mm} \mathrm{mrad}, \sigma_{\gamma_{o}} / \gamma_{x}=0.8 \times$ $10^{-4}, I_{0}=3.4 \mathrm{kA}$, and consider a TOPAS laser [16] with $\lambda_{L}=2200 \mathrm{~nm}$ and $P_{L} \approx 6 \mathrm{GW}$ for energy modulation. We choose to energy modulate the electrons at $2 \mathrm{GeV}$ and consider a wiggler magnet with 10 periods, $\lambda_{w}=16 \mathrm{~cm}$, and $K_{w}=29$ (peak magnetic field $\sim 2 \mathrm{~T}$ ). We note that the energy modulation at low energy is more convenient than at high energy because of the greater length of the wiggler required at higher energy. However, if a wiggler with just one period is the goal, as in the application considered later, then the energy modulation at the end of the linac can be a preferable option. An additional advantage from the energy modulation at low energy is the small energy spread induced in the electron beam by the wiggler magnet itself. 

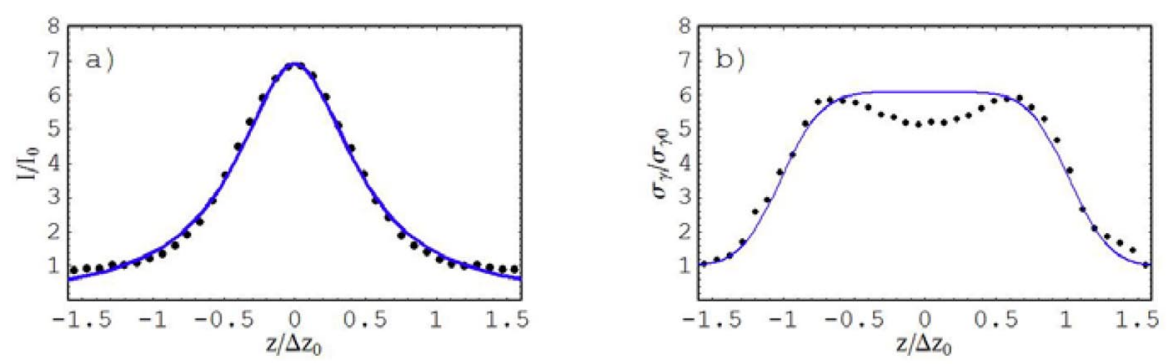

FIG. 3. (Color) Normalized peak current (a) and energy spread (b) versus distance within the microbunch. The dots show computer simulation results while the solid line was obtained with Eqs. (6) and (7).

From Eq. (1) we obtain $\Delta \gamma_{w} / \gamma_{x}=6.4 \times 10^{-4}$ and $B=$ 8. For this energy modulation, a chicane with $R_{56} \approx$ $0.5 \mathrm{~mm}$ provides the required bunching at the laser wavelength. Then, using Eqs. (6) and (7) we plot $I(z) / I_{0}$ and $\sigma_{\gamma}(z) / \sigma_{\gamma 0}$ in Fig. 3 .

Finally, we consider the SASE process in an undulator with $\lambda_{u}=3 \mathrm{~cm}$ and $K_{x}=3.7$ producing x-ray radiation at $\lambda_{x}=0.15 \mathrm{~nm}$. For the Pierce parameter $\rho$ [17] we calculate $\rho \approx 8 \times 10^{-4}$ for the part of the electron beam inside of the WS and $\rho \approx 4 \times 10^{-4}$ for the part of the electron beam outside of the WS. These results indicate a significant reduction of the gain length within the WS. For a more accurate account for the gain length we use a 3D model from Ref. [18] and the fitting formula derived there. We optimize the electron beam focusing in the undulator and find that a beta function of $\beta \approx 6 \mathrm{~m}$ yields the shortest amplitude gain length of $\hat{M}_{G} \cong 115^{1}$ for microbunches inside of the WS and almost 4 times longer gain length for electrons outside of the WS. Then we calculate $M_{G}(z)$ for various pairs of $I(z), \sigma_{\gamma}(z)$ near the peak of the microbunch intensity and plot it in Fig. 4. We note that without ESASE the optimal beta function is $\beta \approx 20 \mathrm{~m}$ and the shortest gain length is $\hat{M}_{G} \cong 275$, i.e., almost 2.5 times longer than with ESASE. We also note that ESASE allows to reach saturation in the $\mathrm{x}$-ray emission at wavelengths shorter than $0.15 \mathrm{~nm}$ down to $0.07 \mathrm{~nm}$, at which point the gain length in the WS becomes as long as the gain length without ESASE at $\lambda_{x}=0.15 \mathrm{~nm}$. Using ESASE one can also pursue the other goal and relax the requirement on the beam emittance to $2.7 \mathrm{~mm}$ mrad, at which point again the gain length in the WS becomes as long as the gain length without ESASE and at the emittance of $1.2 \mathrm{~mm}$ mrad.

We estimate the initial microbunching at wavelength $\lambda_{x}=0.15 \mathrm{~nm}$ (not to be confused with the microbunching at $2200 \mathrm{~nm}$ discussed previously) from shot noise: $b_{0}=$ $\sqrt{e c / 9 I_{0} \mathrm{e} a B \hat{M}_{G} \lambda_{x}} \approx 1.5 \times 10^{-4}$ and calculate that approximately eight gain lengths will be needed to reach

\footnotetext{
${ }^{1}$ An increase of the beta function to $12 \mathrm{~m}$ only marginally increases the gain length to $\hat{M}_{G} \cong 125$.
}

saturation with an assumed maximum bunching $b=0.5$. Then, using Eq. (8), we find that the pulse duration for a single $\mathrm{x}$-ray micropulse $\Delta \tau \approx 200$ asec.

We estimate the peak power at saturation $P_{0} \approx 230 \mathrm{GW}$ using the fitting formula from Ref. [19]: $P_{0} \approx$ $P_{\text {beam }} /\left(8 \pi \hat{M}_{G}\right)$, where $P_{\text {beam }}$ is the beam power. The average power over the entire sequence of the $\mathrm{x}$-ray micropulses is smaller by a duty factor $\lambda_{L} / c \Delta \tau \approx 30$.

The above discussed technique can also be used for generation of attosecond x-ray pulses. Similar to [8,9], we employ interaction of electrons in the short wiggler magnet with a few-cycle laser pulse with a stabilized carrier wave phase. Laser systems producing pulses with a $5 \mathrm{fsec}$ FWHM at $800 \mathrm{~nm}$ wavelength have been demonstrated elsewhere [3-5,20,21]. This pulse with $\sim 0.2 \mathrm{~mJ}$ pulse energy interacting with electrons in a one-period wiggler magnet produces energy modulation in the WS with a relatively rapid variation of the modulation amplitude as shown in Fig. 5(a). This causes a variation of the bunching efficiency of electrons at $800 \mathrm{~nm}$ wavelength in the following dispersive magnetic chicane and formation of the modulation of the electron peak current shown in Fig. 5(b). Here the central peak is approximately 1.5 times higher the neighboring peaks and, therefore, the SASE gain length there is $\sim 14 \%$ shorter. After the eight gain lengths

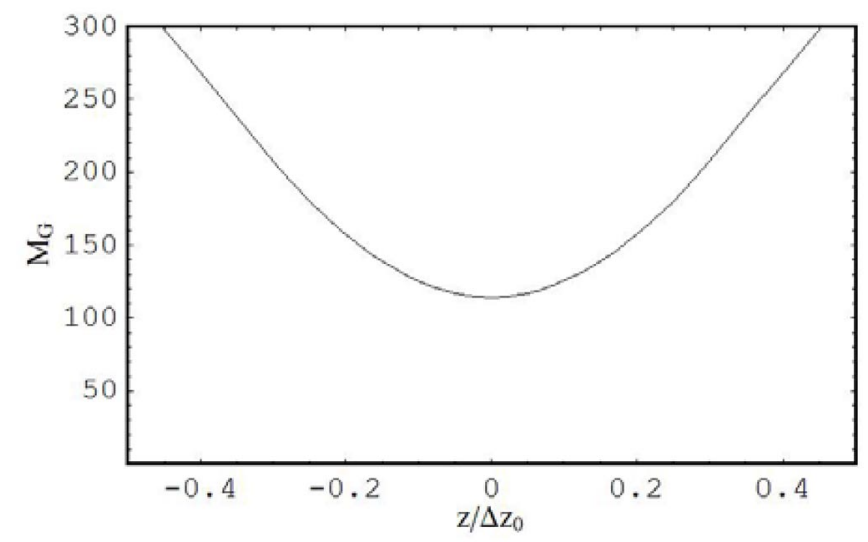

FIG. 4. Gain length at various distances within the microbunch calculated using fitting formula from Ref. [18]. 

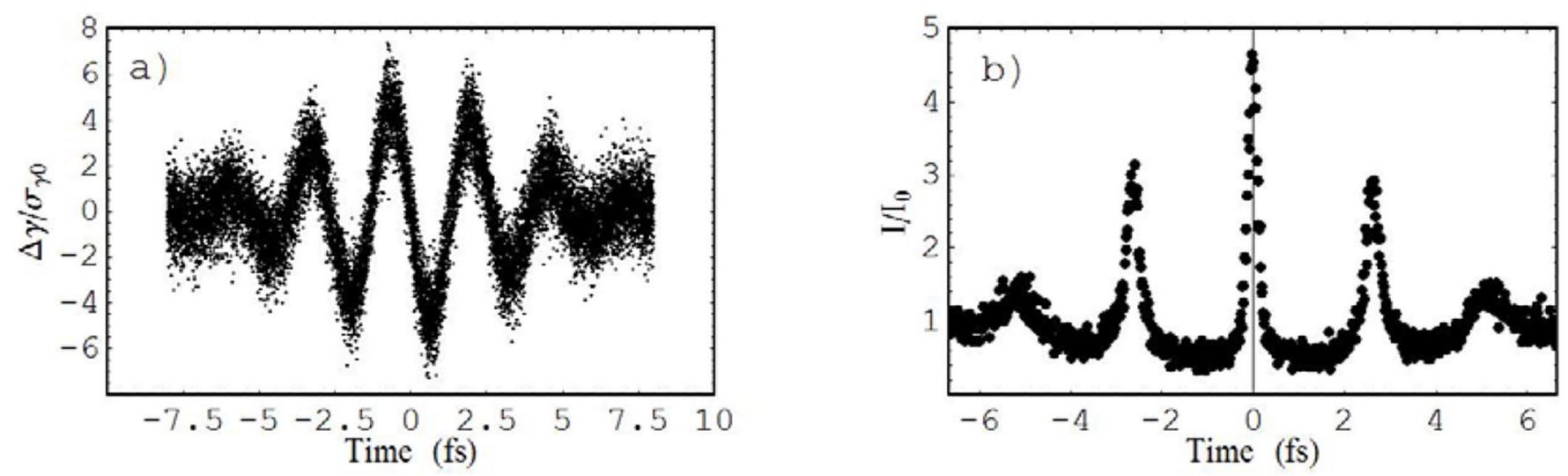

FIG. 5. Energy and peak current modulations produced in interaction with a few-cycle laser pulse. Only a part of the electron bunch affected by the interaction is shown.

this small difference creates a significant difference in the output signal where the radiation from the central peak dominates the radiation from the neighboring peaks. A contribution to the signal from the electrons outside of the WS is negligible because of a much longer SASE gain length there. Thus, we obtain a solitary $x$-ray pulse perfectly synchronized to the modulating laser pulse with approximate pulse width of 200 asec as defined above.

In summary, we have demonstrated that electron peak current can be significantly increased by interaction of the electron beam with a high peak power laser pulse in a wiggler magnet prior to entering a SASE FEL. This shortens the FEL gain length and increases the peak power of $x$ ray radiation which comes out from the FEL as a sequence of $\mathrm{x}$-ray micropulses. Each micropulse in this sequence is nearly temporally coherent and Fourier transform limited and has width on the order of a few hundred attoseconds. However, the carrier phase of the $\mathrm{x}$-ray wave is not stable and changes randomly from one micropulse to the next. The duration of the entire sequence of micropulses can be controlled by adjusting the duration of the optical laser pulse and the number of active wiggler periods. In effect it can be made as short as a single x-ray micropulse by employing a few-cycle laser pulse and a wiggler magnet with one period. There is absolute synchronization between the laser pulse and the x-ray signal, which can be used in pump-probe experiments.

Following the original proposal described in this paper, more studies were carried out and summarized in [22]. A particular consideration there was given to collective effects related to a propagation of the electron microbunches with a high peak current through the undulator with a narrow-gap vacuum chamber. It was demonstrated, albeit preliminary, that those effects do not seriously degrade the performance of the ESASE. In the other development, an idea of generation of attosecond x-ray pulses briefly described here was further advanced in [23].

\section{ACKNOWLEDGMENTS}

This work was supported by the Director, Office of Science, of the U.S. Department of Energy, under Contract No. DE-AC03-76SF00098. Useful discussions with J. Corlett, P. Emma, W. Fawley, Z. Huang, G. Penn, J. Wurtele, and M. Zolotorev are gratefully acknowledged.

[1] C. Pellegrini, Nucl. Instrum. Methods Phys. Res., Sect. A 475, 1 (2001).

[2] See, e.g., Report No. SLAC-R-521, 1998; Report No. DESY 2001-011, 2001.

[3] M. Hentchel, R. Kienberger, Ch. Spielmann, G. A. Reider, N. Milosevic, T. Brabec, P. Corkum, U. Heinzmann, M. Drescher, and F. Kausz, Nature (London) 414, 509 (2001).

[4] M. Drescher, M. Hentschel, R. Kienberger, M. Uiberacker, V. Yakovlev, A. Scrinzi., Th. Westerwalbesloh, U. Kleineberg, U. Heinzmann, and F. Kausz, Nature (London) 419, 803 (2002).

[5] R. Kienberger, E. Goulielmakis, M. Uiberacker, A. Baltuska, V. Yakovlev, F. Bammer, A. Scrinzi, Th. Westerwalbesloh, U. Kleineberg, U. Heinzmann, M. Drescher, and F. Kausz, Nature (London) 427, 817 (2004).

[6] M. Zolotorev, Nucl. Instrum. Methods Phys. Res., Sect. A 483, 445 (2002).

[7] E. L. Saldin, E. A. Schneidmiller, and M. V. Yurkov, Opt. Commun. 212, 377 (2002).

[8] A. A. Zholents and W. M. Fawley, Phys. Rev. Lett. 92, 224801 (2004).

[9] E. L. Saldin, E. A. Schneidmiller, and M. V. Yurkov, Opt. Commun. 237, 153 (2004); Opt. Commun. 239, 161 (2004).

[10] A. A. Zholents, W. Wan, and M. Zolotorev, in Proceedings of the 2001 Particle Accelerator Conference, Chicago, IL (IEEE, Piscataway, NJ, 2001), p. 723.

[11] W. B.Colson and P. Elleaume, Appl. Phys. B 29, 101 (1982). 
[12] W. Colson, Laser Handbook (North-Holland, Amsterdam, 1990), Vol. 6, Chap. 5; J. B.Murphy and C. Pelligrini, Laser Handbook, Chap. 2.

[13] N. A. Vinokurov and A. N. Skrinsky, Institute of Nuclear Physics Report No. INP 78-88, 1978.

[14] M. Abromowitz and I. Stegun, Handbook of Mathematical Formulae, Applied Mathematic Series No. 55 (National Bureau of Standards, Washington, DC, 1964).

[15] R.Bonifacio, L. De Salvo, P. Pierini, N. Piovella, and C. Pellegrini, Phys. Rev. Lett. 73, 70 (1994).

[16] http://www.lightcon.com/lc/scientific/topas.htm

[17] R. Bonifacio, C. Pellegrini, and L. M. Narducci, Opt. Commun. 50, 373 (1984).

[18] M. Xie, Nucl. Instrum. Methods Phys. Res., Sect. A 445, 59 (2000).
[19] K.-J. Kim and M. Xie, Nucl. Instrum. Methods Phys. Res., Sect. A 331, 359 (1993).

[20] T. Brabec and F. Krausz, Rev. Mod. Phys. 72, 545 (2000).

[21] B. Schenkel, J. Biegert, U. Keller, C. Vozzi, M. Nisoli, G. Sansone, S. Stagria, S. De Silvestri, and O. Svelto, Opt. Lett. 28, 1987 (2003).

[22] A. A. Zholents, W. M. Fawley, P. J. Emma, Z. Huang, G. Stupakov, and S. Reiche, in Proceedings of the 2004 Free Electron Laser Conference (FEL2004), Trieste, Italy, http://www.JACoW.org, p. 582.

[23] A. A. Zholents and G. Penn, LBNL Technical Report No. LBNL-56773, 2005, http://www-library.lbl.gov/docs/ LBNL/567/73/PDF/LBNL-56773.pdf 